\title{
In vivo veritas: the continuing importance of discoveries in complex biosystems
}

\author{
C G A Persson
}

\begin{abstract}
The common belief that reductive biological sciences - for example, molecular biology and cellular chemistry - will write the book of revelation of all future antiasthma drugs is at variance with the demonstrated importance of discoveries in complex in vivo systems.

(Thorax 1996;51:441-443)
\end{abstract}

Keywords: drug discovery, asthma pathophysiology.

Current developments provide medical research with powerful analytical tools and easily accessible isolated cell methods. Molecular biology, cellular chemistry, and other "reductive sciences" are thus set on course to find causes and cures of capricious and complex conditions such as asthma. Increasingly, as an abundance of new data accumulates, there is recognition of a need for "integrative physiology". For this latter science the agenda is set; experiments will confirm a role for all the molecular and cellular biology. This review takes another approach and suggests that original and important discoveries will also be made in complex in vivo systems.

\section{Scientific basis to drug discovery}

When novel molecular aspects of "airway inflammation" are unravelled this will prompt a speedy development of chemicals with blocking, mimicking, or other interaction properties. Specific mediator, cytokine, enzyme, and signal transduction hypotheses are also being continuously tested in asthma. As an example of such a "logic" approach, inhibitors of the actions of leukotrienes ${ }^{1}$ are now becoming available as antiasthma drugs. However, the major remedies in asthma may not fit into this simple scheme.

Pharmacology,

University of Lund

and Preclinical R\&D,

Astra Draco AB,

Lund, Sweden

C G A Persson

Correspondence to: Dr C Persson,

Department of Clinical

Pharmacology, University of

Lund, S-221 85 Lund,

Sweden.
The two antiasthma xanthines - caffeine (figure) and theophylline - are known to antagonise adenosine. Based on this knowledge and with an increasingly strong emphasis on receptor and cell biology data, adenosine has been pro- moted as a mediator of asthma. A few years ago when adenosine antagonism was widely viewed as the mechanism of action of theophylline (and of "future drugs"), in vivo data obtained with another xanthine, enprofylline, indicated that blocking the physiological actions of adenosine should probably be avoided in the treatment of asthma; it had no therapeutic effect but resulted in several side effects to the xanthine drug therapy. ${ }^{2}$ The discovery of enprofylline was based on data generated by heuristic research on the effects of selected xanthine derivatives in complex biosystems. At its appearance the "enprofylline paradox" clearly clashed with the concepts and language developed on the basis of reductive biomedical research on adenosine receptors.

Assyrian clay tablets tell about treatment of chest diseases: "... thou shalt bray Ammi, spread it over thorn fire, let the smoke enter his anus, his mouth and his nostrils". ${ }^{3}$ Underscoring the work of Roger Altounyan, ${ }^{4}$ this old Iraqi remedy may be the only known chromone inhalation prior to the advent of cromoglycate. A scientist, physician, and asthmatic, Altounyan discovered the efficacy of cromoglycate by himself inhaling a series of new chromone compounds.

New clinical and pharmacological aspects of both chromones and xanthines continue to be unravelled. However, the reductive modes of action of these two groups of drugs may remain conjectural.

\section{$\beta$-AGONISTS}

Hyde Salter (figure) was an astute observer and a brave scientist. He advocated "simple reading of nature" and showed obvious aversion when "its place (was) supplied by an unquestioning inheritance and adoption of received notions". Salter discovered many aspects of asthma including the fact that violent emotions, causing the release of "a nervous derivative", could produce instantaneous antiasthmatic effects. ${ }^{5}$ When adrenaline eventually became available it was successfully used as a "vasoconstrictor" in asthma. ${ }^{5}$ These early workers could be said to have done the right thing (discovering the antiasthmatic activity of adrenaline injections) for the "wrong" reason (vasoconstriction). This 


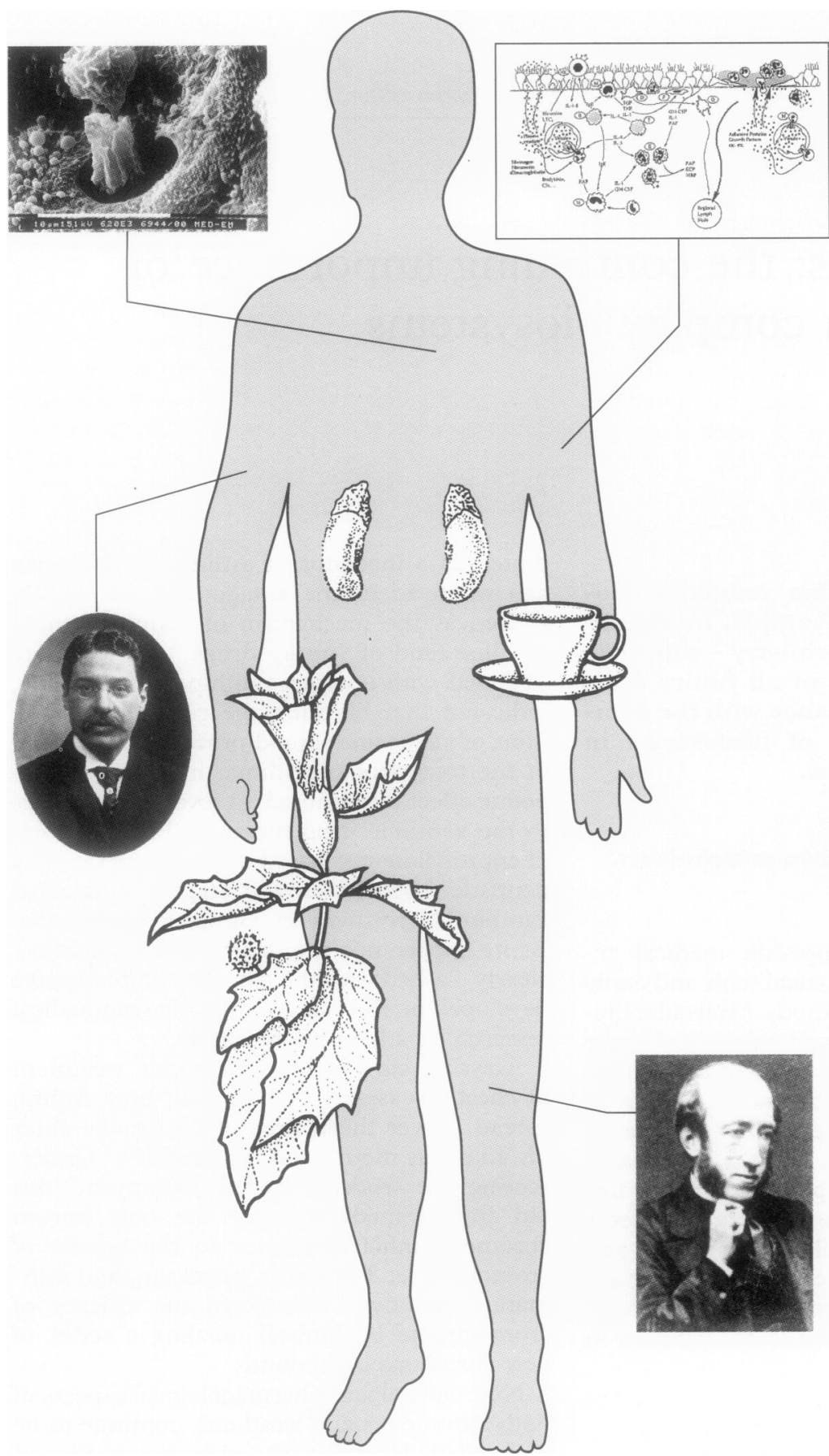

The Datura stramonium plant, the adrenal glands, and the cup of coffee represent the early employment of anticholinergic, hormonal, and xanthine therapies in asthma. Hyde Salter (1823-1870, right) may claim many of the discoveries of the activity of xanthines and sympathomimetics in asthma, while Solomon Solis-Cohen (1857-1948, left) was responsible for discovering much of the antiasthmatic activity of steroids. The

ultrastructural and schematic representations of an inflamed airway mucosa (upper left and right) merely illustrate the superficial and limited aspects of asthma. Astute in vivo observers (followers of Salter, Solis-Cohen and others) should have a continuing role in making important discoveries of both disease abnormalities and therapeutic principles in asthma. (Drawings by Lotta Herold and fonas Erjefält.)

method of making important discoveries continues to be successful. The label "mechanism of action" must frequently be questioned, altered, or remain unknown when interesting in vivo effects of experimental compounds are being observed.

After adrenaline came more selective agents. Several years before the introduction of $\alpha$ and $\beta$ receptors Konzett described the particular properties of isoprenaline, and well before the discovery of $\beta_{1}$ and $\beta_{2}$ receptors researchers in the drug industry with unflinching convictions produced terbutaline and salbutamol. ${ }^{5}$ The importance of generally acknowledged basic research in this field cannot be neglected, but in individual cases - now also including formoterol and salmeterol - the academic theories have not preceded but have rather been dependent on the drug discoveries. The example of "sympathomimetics against asthma" shows that astute, non-dogmatic, almost rebellious researchers may make original important observations.

\section{STEROIDS}

In 1990 Solomon Solis-Cohen (figure) described the antiasthmatic action of maintenance therapy with dried bovine adrenal glands ingested in amounts of 2-6 g daily: "The constant dyspnea first disappeared, the paroxysmal nocturnal attacks became less frequent and less severe. Recovery was not rapid but was continuous." Solis-Cohen's erudite report is probably the first demonstration of the efficacy of oral steroids in asthma. ${ }^{6}$ About 70 years later inhaled beclomethasone dipropionate was introduced, being the first example of topical airway steroid therapy. Budesonide was developed during the 1970 s through structure/activity research with a focus on airway/lung selectivity in vivo. ${ }^{7}$ Such chemical/pharmacological discovery work, similar to the development of novel $\beta$ agonists, is a mixture of basic and goal orientated research in which new, sometimes very unexpected, observations are used to produce a novel drug. As we approach the next century, many of the actions of steroids have been unravelled. Importantly, however, no one has yet established for certain which molecular and cellular action(s) must be produced by a novel drug group to approach the clinical efficacy of the steroids. This scenario, to which we can add the xanthines and the chromones, is perfectly compatible with the possibility that new antiinflammatory drugs to combat asthma and other diseases may be discovered before their proper reductive modi operandi have been delineated.

\section{Therapeutic potential of "reductive" versus "complex" approaches}

It is taken for granted that molecular biology is applied secondary to cell biological studies - that is, cellular mechanisms and functions must first be well assessed. To take this logic one step further, I suggest that the in vivo physiology/pathophysiology must be right (if not known it must at least be part of our test system) before we resort too much to the reductive sciences, or the reductive approach may involve the risk of exploring and explaining phantom phenomena.

My own acquaintance with this potential problem concerns the role of adenosine antagonists in asthma (mentioned above). Another aspect of some importance to drug development is the finding that neurogenic exudative inflammation may not exist in human 
airways in vivo, although it is a dominating mechanism in the airways of commonly employed experimental animals. ${ }^{8}$ Of physiological relevance are in vivo data which show that the mucosal barrier may be abnormally tight in allergic diseases of the airways. ${ }^{9}$ This latter observation contrasts with the common view of increased mucosal permeability in inflammation of the airways (which may also have been "explained" at reductive levels). Since the epithelial lining is the main contributor to mucosal tightness, the assumption that the permeability should be increased in desquamative diseases of the airways has been quite logical. However, the possibility of a speedy creation of barriers in vivo must now be taken into consideration. ${ }^{10}$ The discoveries here include prompt and quick epithelial restitution as well as further data suggesting potential involvement of the latter process in causing the pathophysiology, the leucocyte pathology, and the structural changes in asthmatic airways. ${ }^{1011}$ Again, the in vivo picture may differ radically from current concepts which are built on cell culture data. (Although exceedingly important techniques, the nontranslatability of generated data into the in vivo model may be a growing problem in studies using cell cultures.)

\section{Conclusions}

The medical history of antiasthmatic treatment may give some insight into the nature of research into drug discoveries. Breakthough observations leading to the development of novel therapeutics can obviously be made in complex whole organ test systems. Original observations of effects or constellation of effects can thus be used to develop innovative drugs before the acknowledged theoretical research has even been able to predict such possibilities. (The antigastric secretion drug omeprazol is an example of this kind of discovery.) Astute observations on functions and processes in vivo may equally be starting points for the unravelling of new facts about the disease. "Hard data on in vivo functions first, soft data on explanatory mechanisms later."
In addition to the ability to observe, in vivo work demands many skills which take time to acquire. The successful experimental setup may involve long hours of preparation and observation, and variability due to unknown factors may appear. Refinement and development of the methodology never ends, particularly when the aim is to create test systems which will mimic complex features of the "wild" asthma disease. The effort is worthwhile, however, because such test systems in experimental animals and humans are fertile fields for original and important observations. The potential for discoveries here should only increase with increasing knowledge of relevant reductive mechanisms. The increasing availability of interesting molecules is also a significant asset. However, the crucial work is that which is carried out by enquiring in vivo researchers, a species that is now threatened by extinction. Indeed, failure to understand the importance of exploratory in vivo approaches may be a major factor in the general slowdown in drug discovery during the last few decades. ${ }^{12}$

1 Dahlén S-E, Hedqvist P, Hammarström S, Samuelsson B. Leukotrienes are potent constrictors of human bronchi. Nature 1980:288:484-6.

2 Persson CGA, Pauwels R. Pharmacology of antiasthma xanthines. In: Page CP, Barnes P, eds. Pharmacology of asthma. Berlin: Springer, 1991:207-25.

3 Mann RD. Modern drug use. An inquiry on historical principles. Lancaster: MTP, 1984

4 Altounyan REC. Review of clinical activity and mode of action of sodium cromoglycate. Clin Allergy 1980;10:481-

5 Persson CGA. On the medical history of xanthines and other remedies for asthma: a tribute to $\mathrm{H}$. H. Salter. Thorax 1985;40:881-6.

6 Persson CGA. Airway microcirculation, epithelium and glucocorticoids. In: Schleimer R, Busse W, O'Byrne P, eds. Topical glucocorticoids in asthma - mechanisms and clinical Topical glucocorticoids in asthma-mechanist
action. New York: Dekker, 1995 (in press).

7 Brattsand R, Selroos O. Current drugs for respiratory diseases. Glucocorticoids. In: Page CP, Metzger WJ, eds. eases. Glucocorticoids. In: Page CP, Metzger WJ, eds.
Drugs and the lung. New York: Raven Press, 1994:101-220.

8 Prugs and the lung. New York: Raven Press, 1994:10 Wollmer $P$, Alkner U, et al. The use of the nose to study the inflammatory response of the respiratory tract. Thorax 1992;47:992-1000.

9 Greiff L, Wollmer P, Svensson C, Andersson M, Persson CGA. Effect of seasonal allergic rhinitis on airway mucosal absorption of chromium-51 labelled EDTA. Thorax 1993; 48:648-50.

10 Erjefält JS, Erjefält I, Sundler F, Persson CGA. In vivo restitution of airway epithelium. Cell Tiss Res 1995;281: 305-16.

11 Erjefält JS, Sundler F, Persson CGA. Eosinophils, neutrophils, and venular gaps in the airway muscosa at epithelial removal-restitution. Am f Respir Crit Care Med 1995 (in press.)

12 Wurtman RJ, Bettiker RL. The slowing of treatment discovery, 1965-1995. Nature Med 1995;1:1122-5. 